\title{
Coordinated regulation of AMPK activity by multiple elements in the $\alpha$-subunit
}

Cell Research (2013) 23:1237-1240. doi:10.1038/cr.2013.121; published online 3 September 2013

\section{Dear Editor,}

The AMP-activated protein kinase (AMPK) is the key energy sensor in response to various stresses that decrease cellular ATP levels, including low glucose, hypoxia, ischemia and heat shock. Recently, evidence has suggested that AMPK also plays critical roles in coupling the cellular bio-energetic state to various physiological processes, such as tumor suppression, cell polarity, life span, circadian clock, gene transcription and autophagy. Thus, AMPK represents a potential drug target for metabolic disorders and cancer treatment $[1,2]$.

AMPK is a heterotrimeric protein kinase composed of one catalytic $(\alpha)$ and two regulatory ( $\beta$ and $\gamma$ ) subunits (Figure 1A). The $\alpha$-subunit contains an $\mathrm{N}$-terminal kinase domain, followed by an autoinhibitory domain (AID), a regulatory linker region ( $\alpha$-linker) and a $\mathrm{C}$-terminal $\beta$ / $\gamma$-subunit interacting domain $(\alpha$-CTD). Phosphorylation of a conserved threonine in the kinase domain (Thr172 in rat $\alpha 1)$ by upstream protein kinases is the prime activation step of AMPK, while the adjacent AID directly binds to the kinase domain and inhibits its catalytic activity [3]. The heterotrimeric core structures of the yeast and mammalian AMPKs, including the entire $\gamma$-subunit and the interacting fragments of the $\alpha$ - and $\beta$-subunits, have revealed that the $\mathrm{C}$-terminal domain of the $\beta$-subunit $(\beta$-CTD) serves as a bridge between the $\alpha$-CTD and the $\gamma$-subunit and that the $\gamma$-subunit adopts a pseudosymmetric conformation comprising four tandem cystathionine $\beta$-synthase (CBS1-4) motifs for adenine nucleotide binding [4]. Binding of AMP and ADP to the $\gamma$-subunit has activating effects in protecting AMPK against dephosphorylation and/or promoting its phosphorylation $[5,6]$. In addition, raising the cellular AMP level will allosterically enhance the activity of mammalian AMPK that has been primarily activated by upstream kinases. Therefore, a central issue pertaining to the regulation of AMPK lies in how AMP binding to the $\gamma$-subunit ultimately regulates the kinase activity in the $\alpha$-subunit.

Although the $\gamma$-subunit contains four potential nucleotide binding sites (1-4), site 2 in mammalian AMPKs possesses a key Asp-to-Arg substitution and remains unoccupied even in the presence of high concentrations of AMP or ATP $[4,7]$. The other three sites $(1,3$ and 4$)$ on the $\gamma$-subunit appear to reversibly bind three AMP or two ATP molecules, which results in substantial conformational changes and distinct activities of AMPK [7]. Our structure-based mutagenesis studies have demonstrated that two of the three nucleotide-binding sites, $\gamma$-site 3 and $\gamma$-site 4, are important for AMPK allosteric activation. Xiao et al. [8] reported the structure of an active AMPK heterotrimer, comprising the kinase domain, AID and the regulatory $\alpha$-linker of the $\alpha$-subunit in addition to the core fragment, which revealed additional intersubunit interactions involving the activation loop of the kinase domain and a small segment within the $\alpha$-linker. However, in the same study, it was concluded that mammalian AMPK does not contain an AID domain, inconsistent with our results [3]. We found that the amino acid sequence of the AID and $\alpha$-linker region in that AMPK structure was incorrectly assigned due to the poor electron density [9]. We have thus rebuilt the model and determined the structures of two isolated mammalian AID domains, which affirms the universal presence of AID (Figure 1B and Supplementary information, Figure S1). More importantly, we identified the $\alpha$-RIM (regulatory subunit interacting motif) sandwiched in between $\gamma$-site 3 and the induced $\beta$-loop (Supplementary information, Figure S2), and we also determined the essential role of $\alpha$-RIM in AMPK allosteric regulation upon AMP binding [9].

Intriguingly, the $\alpha$-linker segment prior to the $\alpha$-RIM reaches the other side of the induced $\beta$-loop and then packs against the non-bound site 2 on the $\gamma$-subunit (Figure 1B). This $\alpha$ - and $\gamma$-subunit interface is mediated by a novel $\alpha$-subunit motif containing the last turn of AID helix $\alpha 3$ and the following flexible loop (rat $\alpha 1332$ 342 ). According to the linear sequence of the $\alpha$-subunit, we hereafter refer to this new segment as $\alpha$-RIM1 and rename the previously identified $\alpha$-RIM as $\alpha$-RIM 2 . At the interface between $\alpha$-RIM1 and $\gamma$-site 2 , the aromatic residues $\alpha$ Phe340 and $\alpha$ Tyr341 interact with $\gamma$ Lys 173, 
$\gamma$ Phe174 and $\gamma$ Leu177 from the $\gamma$-Helix 7; in turn, the hydrophobic $\gamma$ Phe 178 inserts between $\alpha$ Ile 333 and $\alpha \mathrm{Met334}$ (Figure 1C). In addition, the highly conserved $\gamma \operatorname{Arg} 170$, which is believed to be the main determinant of the non-bound site 2, further strengthens this interface by participating in multiple hydrophilic interactions with the main chains of $\alpha$ Phe340 in $\alpha$-RIM1 and $\gamma$ Leu 40 and $\gamma$ Pro42 from an N-terminal segment of the $\gamma$-subunit, while the main chains of $\gamma \mathrm{Asp} 39, \gamma \mathrm{Thr} 43$ and $\gamma \operatorname{Ser} 44$ from this $\gamma$-subunit segment form hydrogen bonds with both the side and main chains of $\alpha$ Tyr341 in $\alpha$-RIM1. Therefore, both the $\gamma$-Helix 7 at site 2 and the spatially

A

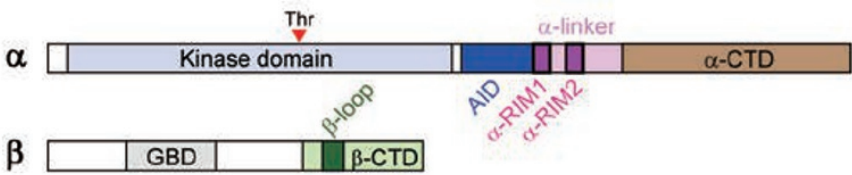

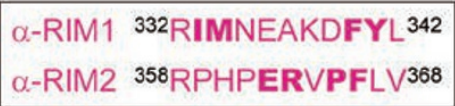

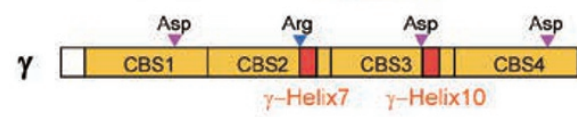

B

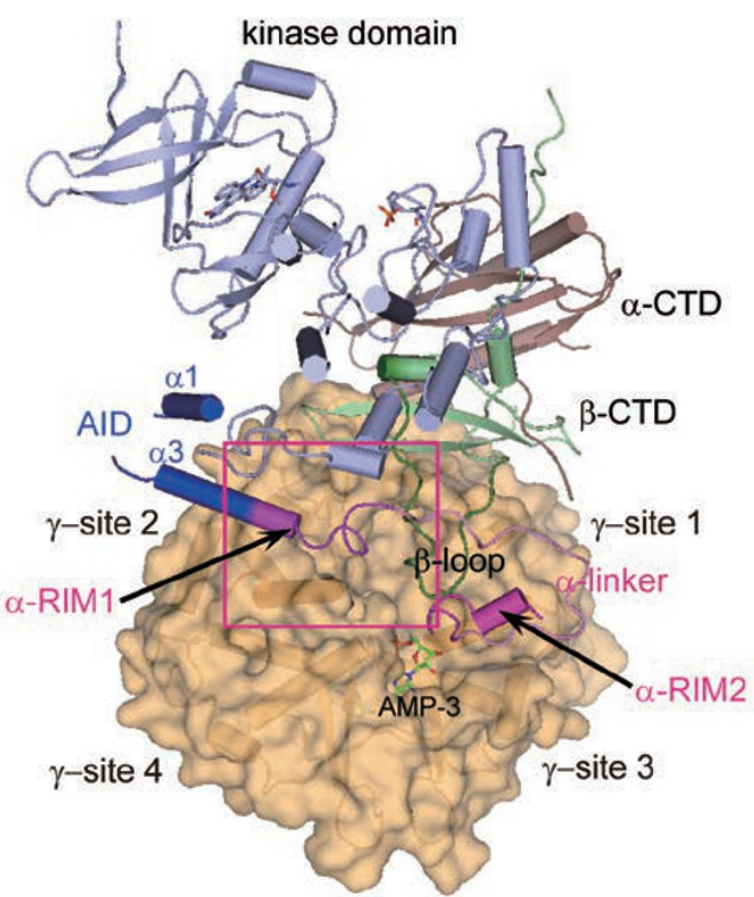

$E$

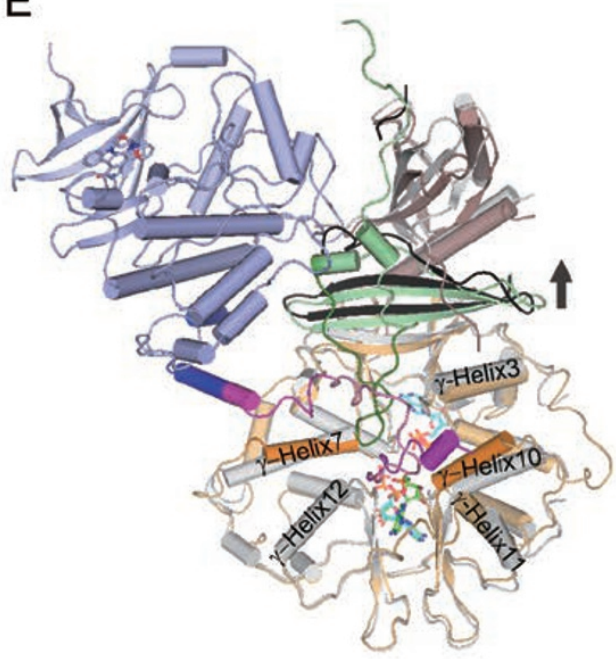

F

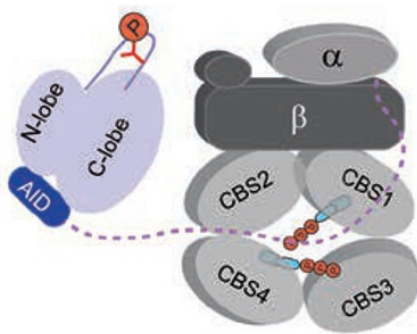

ATP-bound
C
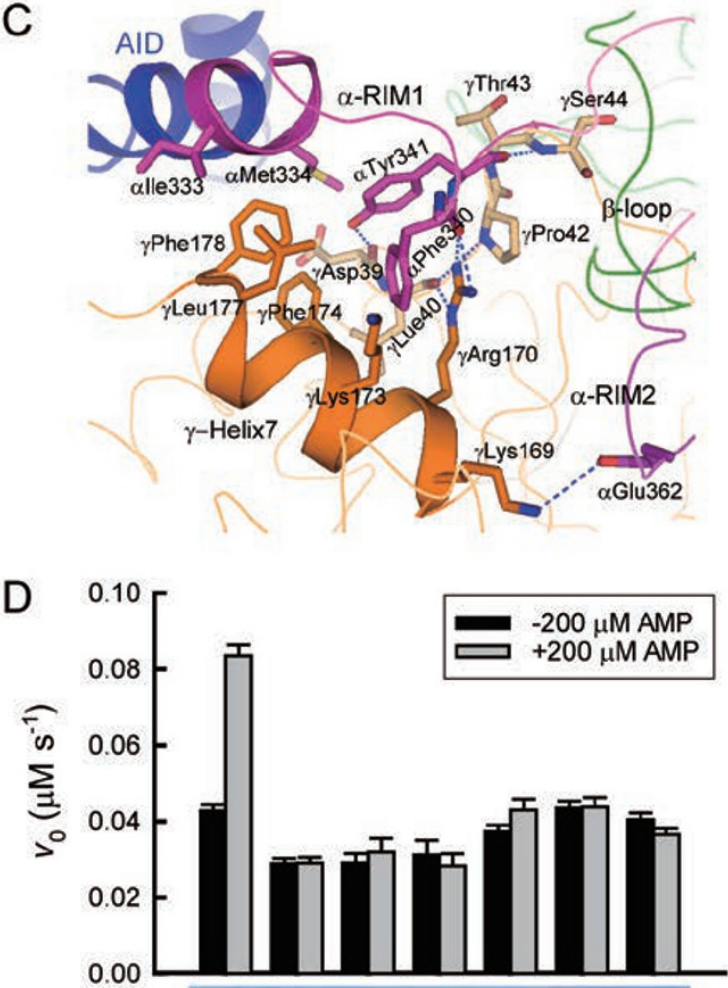

anti-pThr172
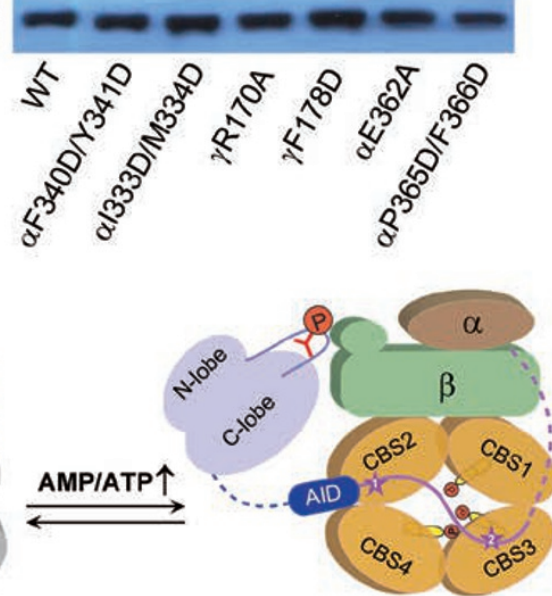

AMP-bound 
Figure 1 AMPK regulation via recruitment of $\alpha$-RIM1 to $\gamma$-subunit site 2. (A) Schematic diagram of the $\alpha \beta \gamma$-heterotrimeric AMPK. The color codes are as follows: kinase domain (light blue), AID (blue), $\alpha$-linker (pink), $\alpha$-RIM1/2 (magenta), $\alpha$-CTD (brown), $\beta$-CTD (green), $\gamma$-subunit (light orange). The sequences for $\alpha$-RIM1 and $\alpha$-RIM2 in rat $\alpha 1$ are indicated on the right. (B) Graphical representations of the rebuilt active AMPK [9]. The color scheme follows the schematic diagram in A with AMP highlighted as green stick. The traceable helices $\alpha 1$ and $\alpha 3$ of AID are indicated. (C) Contacts between $\alpha$-RIM1 and $\gamma$-site 2 . The interacting residues were highlighted as sticks, and the hydrogen bonds were indicated by blue dashed lines. (D) Both $\alpha$-RIM1 and $\alpha$-RIM2 play significant roles in the allosteric activation of AMPK. The kinase assay for AMPK was performed in the presence of $7 \mathrm{nM}$ AMPK (wild type or mutants), $200 \mu \mathrm{M}$ SAMS, $1 \mathrm{mM} \mathrm{ATP}$ and $10 \mathrm{mM} \mathrm{MgCl} \mathrm{m}_{2}$, with or without the addition of $200 \mu \mathrm{M}$ AMP (mean $\pm \mathrm{SD}, \mathrm{n}=3$ ). The bottom panel shows the phosphorylation levels of all AMPK proteins detected by western blotting using a specific antibody against phosphorylated AMPK (anti-pThr172). (E) Structural comparison of the rebuilt active AMPK [9] with the ATP-bound core structure (PDB code: 4EAK) [7]. The color scheme for the ATP-bound core is as follows: $\alpha$ - and $\gamma$-subunits in grey, $\beta$-subunit in black and two ATP molecules at sites 1 and 4 in cyan. (F) Allosteric regulation of mammalian AMPK by AMP/ATP. The $\gamma$-subunit of AMPK adopts an inactive, ATP-bound conformation (left) when the cellular AMP concentration is low. The $\alpha$-linker is likely flexible and extended (dotted line), and AID directly binds to the kinase domain and inhibits the AMPK activity. Under energy stress, the $\gamma$-subunit is bound by AMP (right), undergoes substantial conformational changes and recruits both $\alpha$-RIM1 and $\alpha$-RIM2. The constrained $\alpha$-linker pulls AID away from the kinase domain, and the activity of AMPK is eventually stimulated.

adjacent $\gamma$-subunit segment (rat $\gamma 1$ 39-44) play important roles in recruiting the newly identified $\alpha$-RIM1. On close inspection, we also found additional hydrophobic interactions involving the previous $\alpha$-RIM2 (Supplementary information, Figure S3). The $\alpha \operatorname{Pro} 365$ and $\alpha \mathrm{Phe} 366$, which follow the two important charged residues $\alpha \mathrm{Glu} 362$ and aArg363 [9], nestle into a hydrophobic pocket formed by six residues at $\gamma$-site 3 ( $\gamma$ Phe243, $\gamma$ Ile246, $\gamma$ Asn247, $\gamma$ Ala250, $\gamma$ His 267 and $\gamma$ Val64). Notably, the aromatic ring of $\gamma \mathrm{Phe} 243$, the residue preceding the indispensable and highly conserved $\gamma$ Asp244, also stabilizes the ribose ring of AMP bound at site 3. Taken together, two tandem $\alpha$-subunit motifs, the $\alpha$-RIM1 and the previously defined $\alpha$-RIM2, bind to site 2 and site 3 on the $\gamma$-subunit, respectively.

To assess the importance of the aforementioned intersubunit interactions, we generated a series of point mutations on the rat $\alpha 1 \beta 1 \gamma 1$-holoenzyme and examined their effects on AMPK allosteric activation. In the presence of $200 \mu \mathrm{M}$ AMP, the activity of the wild-type AMPK was stimulated by $\sim 2$-fold (Figure 1D). In contrast, mutating the key $\alpha$-RIM1 residues largely abolished the AMP dependence as observed in the mutants in which the hydrophobic pairs of interacting residues were replaced with charged residues $(\alpha \mathrm{F} 340 \mathrm{D} / \mathrm{Y} 341 \mathrm{D}$ and $\alpha \mathrm{I333D} / \mathrm{M} 334 \mathrm{D})$. Similarly, the $\gamma$-site 2 mutants ( $\gamma$ R170A and $\gamma$ F178D) no longer responded to the increase in AMP concentration. We also simultaneously substituted the two hydrophobic residues in the $\alpha$-RIM2, and the AMP dependence of the $\alpha \mathrm{P} 365 \mathrm{D} / \mathrm{F} 366 \mathrm{D}$ mutant was completely abolished as previously observed in other $\alpha$-RIM2 mutants, including $\alpha E 362 \mathrm{~A}[9]$. The independence of these mutant AMPKs on changes in AMP concentration clearly demonstrates that both $\alpha$-RIM1 and $\alpha$-RIM2 play important roles in the allosteric regulation of AMPK activity.
Significantly, in the vertebrate AMPK homologs, 7 out of the 8 important interacting residues in the new $\alpha$-RIM1 and previous $\alpha$-RIM2 are identical, and the remaining one is highly conserved (Supplementary information, Figure S1). The interacting $\gamma$-subunit residues are also strictly conserved across vertebrates (Supplementary information, Figure S4). Therefore, various AMPK $\alpha \beta \gamma$ heterotrimers may adopt similar inter-subunit interactions and be activated via a conserved molecular mechanism. By contrast, some of these residues in the $\alpha$-RIM $1 / 2$ and $\gamma$-sites 2 and 3 are not conserved in invertebrate AMPKs, such as those from Caenorhabditis elegans, Arabidopsis thaliana, Saccharomyces cerevisiae and Schizosaccharomyces pombe. This provides a possible explanation for why only vertebrate AMPKs can be allosterically activated by AMP.

The next question is how AMPK senses the change in the cellular AMP/ATP levels. We have shown that the mammalian AMPK core structures in complex with ATP or AMP adopt distinct conformations, in that the $\gamma$-site 3 is free of any nucleotide in the ATP-bound state but bound by AMP in the AMP-bound state [7]. This active AMPK structure in complex with two AMP molecules at $\gamma$-sites 3 and 4 adopts an almost identical conformation to the AMP-bound core structure (Supplementary information, Figure S2), but it exhibits substantial conformational changes around $\gamma$-site 3 compared to the ATPbound structure (Figure 1E). In the ATP-bound structure, the $\alpha$-CTD and $\beta$-CTD, as well as the first $\beta$-strand of the $\gamma$-subunit that forms an inter-subunit $\beta$-sheet with the $\beta$-CTD, collectively move upwards, which would abolish the multiple hydrogen bonds between the main chains of the $\gamma$-subunit N-terminal segment and the $\alpha \operatorname{Tyr} 341$ of $\alpha$-RIM1 (Figure 1E and Supplementary information, Figure S5A). The side chains of $\gamma \operatorname{Arg} 170$ and $\gamma$ Lys 173 at 
$\gamma$-site 2 also shift away from $\alpha$-RIM1, which may disrupt both the hydrophilic and hydrophobic interactions with $\alpha$ Phe340. In addition, the $\gamma$-Helix 7 tilts $\sim 5^{\circ}$ and forms an additional turn at the $\mathrm{C}$-terminus, and the aromatic ring of $\gamma \mathrm{Phe} 178$ thereby rotates almost $180^{\circ}$ and moves away from the hydrophobic side chains on the AID helix $\alpha 3$. Hence, when the $\gamma$-subunit is bound by ATP, most of the interactions between the $\alpha$-RIM1 and the $\gamma$-subunit would also be disrupted. Upon ATP binding, the most significant shift (up to $6 \AA$ ) occurs in $\gamma$-Helix10, which contains the highly conserved Asp244 as well as four of the six residues accommodating the hydrophobic $\alpha$ Pro365 and $\alpha$ Phe366 in $\alpha$-RIM2 (Supplementary information, Figures S5B and S3). The spatially adjacent $\gamma$-Helix11 and $\gamma$-Helix3 also move away from $\alpha$-RIM2; specifically, the imidazole side chain of $\gamma \mathrm{His} 267$ rotates $\sim 180^{\circ}$ and shifts $>5 \AA$. In addition, the $\varepsilon$-amino group of $\gamma$ Lys 169 is pushed away from the key $\alpha$ Glu362 by the dramatically changed $\gamma$ His 297 . Therefore, the $\gamma$-site 3 in the ATP-bound state is distorted and unable to recruit $\alpha$-RIM2. In contrast, AMP binding to the $\gamma$-subunit induces substantial changes and enables recruitment of both $\alpha$-RIM1 and $\alpha$-RIM2 to the $\gamma$-subunit.

Then, why and how would the binding of two RIM motifs ultimately enhance AMPK activity in the $\alpha$-subunit? We suggested previously that the $\gamma$-sites 3 and 4 of mammalian AMPKs are indispensable for AMP-induced allosteric regulation and that the $\gamma$-site 1 has little effect [7]. It is natural that $\alpha$-RIM2 plays an important role in the AMP regulation of AMPK activity because $\alpha$-RIM2 packs against the essential $\gamma$-site 3 in its AMP-bound state [9]. Here we demonstrate that $\alpha$-RIM1, though surprisingly bound to the nucleotide-free $\gamma$-site 2 , has a significant effect on AMPK regulation. As $\alpha$-RIM1 partially overlaps with the AID domain, binding of $\alpha$-RIM1 to the $\gamma$-site 2 would recruit the AID domain to the proximity of the $\gamma$-subunit. More importantly, all the AID side chains that were shown to interact with the kinase domain in the autoinhibited AMPK structure [3] are facing the $\gamma$-subunit, and binding of the kinase domain to AID in the active AMPK conformation would thus cause severe steric clash between the kinase domain and the $\gamma$-subunit (Supplementary information, Figure S6). As revealed in our rebuilt structure of the active AMPK, binding of $\alpha$-RIM1 and $\alpha$-RIM 2 to the AMP-bound $\gamma$-subunit forces the kinase domain to dissociate from AID and pack against the $\beta$-CTD, thereby relieving the inhibitory function of AID.

A full understanding of the regulation of AMPK upon energy stress may facilitate the design of novel therapeutics for metabolic diseases. Together with our previous studies, the structural and mutational analyses demon- strate that the three regulatory elements in the $\alpha$-subunit, AID, $\alpha$-RIM1 and $\alpha$-RIM2, are indispensable for the allosteric activation of AMPK, leading to a more complete understanding of the allosteric regulation of mammalian AMPK (Figure 1F). When the cellular AMP concentration is low, $\alpha$-RIM1 and $\alpha$-RIM2 do not bind to the ATP-bound $\gamma$-subunit. AID directly binds to the kinase domain, thus leading to the autoinhibition of AMPK activity. Under energy stress (increased AMP levels), the $\gamma$-subunit is bound by AMP and undergoes substantial conformational changes. The $\alpha$-RIM1 and $\alpha$-RIM2 are recruited to $\gamma$-site 2 and $\gamma$-site 3 , respectively. The AID domain is thus pulled into the proximity of $\gamma$-site 2 and dissociates from the kinase domain. The free kinase domain then binds to the $\beta$-CTD, and the heterotrimeric AMPK holoenzyme adopts a more compact, fully activated conformation [10]. Following this model, molecules that can stabilize the interactions between $\alpha$-RIM1/2 and $\gamma$-sites $2 / 3$ or disrupt those between AID and the kinase domain would retain AMPK in a stimulated state.

\section{Acknowledgments}

This work was supported by the National Natural Science Foundation of China (31130062), the National Key Basic Research Program of China (2013CB530603) and Tsinghua University (20121080028).

\section{Feng-Jiao Xin ${ }^{1, *}$, Jue Wang ${ }^{1, *}$, Rong-Qing Zhao ${ }^{1}$, Zhi-Xin Wang ${ }^{1}$, Jia-Wei Wu ${ }^{1}$}

${ }^{I}$ MOE Key Laboratory of Protein Science and Tsinghua-Peking Center for Life Sciences, School of Life Sciences, Tsinghua University, Beijing 100084, China

*These two authors contributed equally to this work.

Correspondence: Jia-Wei Wu

Tel: 86-10-62789387; Fax: 86-10-62792826

E-mail: jiaweiwu@mail.tsinghua.edu.cn

\section{References}

1 Steinberg GR, Kemp BE. Physiol Rev 2009; 89:1025-1078.

2 Hardie DG. Biochem Soc Trans 2011; 39:1-13.

3 Chen L, Jiao ZH, Zheng LS, et al. Nature 2009; 459:1146-1149.

4 Kemp BE, Oakhill JS, Scott JW. Structure 2007; 15:1161-1163.

5 Hardie DG, Carling D, Gamblin SJ. Trends Biochem Sci 2011; 36:470477

6 Oakhill JS, Scott JW, Kemp BE. Trends Endocrinol Metab 2012; 23:125-132.

7 Chen L, Wang J, Zhang YY, et al. Nat Struct Mol Biol 2012; 19:716718 .

8 Xiao B, Sanders MJ, Underwood E, et al. Nature 2011; 472:230-233.

9 Chen L, Xin FJ, Wang J, et al. Nature 2013; 498:E8-E10.

10 Zhu L, Chen L, Zhou XM, et al. Structure 2011; 19:515-522.

(Supplementary information is linked to the online version of the paper on the Cell Research website.) 